\title{
Steering Kinematics and Turning Resistance Experimental Investigation of Articulated Rigid Body Vehicles
}

\author{
Aleksander Skurjat \\ Department of Off-road Machine and Vehicle Engineering, \\ Wroclaw University of Technology, \\ I. Łukasiewicza 7/9, 50-374 Wrocław, \\ aleksander.skurjat@pwr.edu.pl
}

Keywords: Off-Road Vehicle, Articulated Body Steering System, Steering Kinematics

\begin{abstract}
Steering kinematics and turning resistance torque depends on geometrical parameters, mass distribution and a type of suspension system of an articulated body steer vehicle with a combination of a ground type. It is important how does a driving system is designed because of its influences on wheels torque while turning. When a vehicle is turning an angle in an articulated joint is rising and the front and rear frame rotate themselves by an angle to the symmetry line when previously situated vehicle forward. In a paper, a method for front and rear frame measuring angles of rotation is presented. There are also results of turning resistances with different mass in articulated wheel loader bucket and two types of ground. Results show, that increasing of a wheeled bucket load influences both steering torque required for turning and changes angles of rotation of the front and rear frame of a vehicle. Steering torque at a first phase is measured to rise to maximum level then decrease even bucket load increase. We can observe that revolution angle of front and rear frame during steering depends strongly on a bucket load, ground type and driving system. Knowledge in rotation angles gives an opportunity to propose a mathematical model for future prediction turning resistance torques.
\end{abstract}

\section{Introduction}

Off-road rigid frames earth working vehicles allows for increased efficiency in construction tasks. There are many difficulties in the proper design of such machines. Most of these vehicles use articulated steering system. Problem with measurements arises when a system is more complex and typically few of coefficients/parameters change their values at the same time. The one of such complex system is articulated hydrostatic steering system of wheeled vehicles with is corresponding with different types of ground. While the vehicle is turning, many parameters like torque arm, oil bulk modulus, a pressure is changing. We have to take into account different ground types too.

A very important disadvantage of off-road vehicles with articulated steering system is low maximum velocity. This is made by snaking phenomena considered as self-path changing due to disadvantages of the steering system. Knowledge how articulated machine steers is crucial in finding equations to calculate and then putting an automatic correction in a path of such vehicle. We can observe (measure) an angle between the front and rear part of a vehicle $\gamma$ very easily by using most types of angle transducers. The angle measured in the steering joint $\gamma$ is a sum of two: course angle of front frame $\gamma_{\mathrm{p}}$ and rear frame $\gamma_{\mathrm{t}}$ and represents an angle of rotation of the front and rear frame. Course angle, in definition, is an angle measured between the velocity vector of the front or rear frame to the line crossing centres of the front and rear axles. Ratios between $\gamma_{p}$ and $\gamma_{\mathrm{t}}$ depend on many situations: travelling velocity, normal reactions on tires (load in a bucket), vehicles geometry, ground type. Ratio calculation between $\gamma_{\mathrm{p}}$ and $\gamma_{\mathrm{t}}$ only from 
geometrical parameters can produce a significant error and no path correction algorithm can be introduced.

In the article, the author presents an experimental method for estimating the rate between the front and rear course angle $\left(\gamma_{p}\right.$ and $\left.\gamma_{t}\right)$ with correlation to the steering angle $(\gamma)$. The measurement system consists of the pressure sensor in a chamber of steering cylinder, articulation sensor and the Earth magnetic field sensor, which are used to measure the course angle of a front frame $\left(\gamma_{\mathrm{p}}\right)$.

A second problem with achieving high velocities of articulated vehicles is made by a low stiffness of the hydraulic system. In previous research of authors, obtained measurement values of steering stiffness were presented with contact or without contact tires to the ground. With the use of torque generation device between the front and rear frames $M_{s}$, steering stiffness is calculated. By the use of the presented measuring system, the author shows forces between ground and tires in function of articulated angle (turning resistance forces) [4]. The results are obtained by the use of steering system designed by the manufacturer. The aim of measurement is to answer, how load and velocity affect turning resistance in universal equations.

\section{Steering kinematics of articulated vehicles}

Steering kinematics of an articulated vehicle at standstill mostly depends on steering system type and geometry. For the vehicles with steering wheels (Ackerman system), a vehicle steers properly when perpendicular lines to the velocity vector of all wheels insect in one point called a turning point. In this case, the moving direction of the vehicle is known and of course with an assumption of a course angle of wheels. Course angle is a result of centrifugal forces, lateral forces etc.

In the case of articulated vehicles, a situation is not identified. The steering system hydraulic actuator makes a rotation of the front and rear frames each another. It is not clear what is a value of turning angle $\left(\gamma_{p}\right.$ and $\left.\gamma_{t}\right)$ of frames to the ground. A theory says that a sum of revolution angles of frames measured to the ground is giving an angle in a steering joint. In real conditions, we can observe two opposed situations. In the first edge situation only one frame rotates (second is fixed), or in a second case, both of them turn the same angle. The result depends on vehicles and steering system geometry, turning resistances, frame masses, tires rolling resistances torques (with a driving system).

Steering system kinematics and turning resistances experimental investigations were performed in the Department of Off-road Machine and Vehicle Engineering, Wroclaw University of Technology. Many research can be found in this field of an experiment [1, 2]. We can observe that when an articulated vehicle is turning at standstill, the middles of centres of differentials $\mathrm{O}_{\mathrm{p}}$ and $\mathrm{O}_{\mathrm{t}}$ get closer to each other - Fig. 1 .

Experimental investigations show, that when turning resistances of a front and rear frame are equal we can observe a symmetrical trajectory $p$ and $t$, and the same angles of rotation $\gamma_{p}$ and $\gamma_{t}$ showed on Fig. 2. The case of inequality of turning resistances results in different $\gamma_{p}$ and $\gamma_{t}$ and even the situation that one frame is fixed showed on Fig. 3. The source of turning resistances torque considering two wheels on driving axle are: rolling resistance torques, braking/driving torques from a drive, tires normal reactions, deformation of a soil. More details considered turning kinematics, specifically forces between tires and a ground and then needed explanations of unused in the article markings and symbols can be found in [1, 2].

Experimental results are giving an answer how does a steering joint angle $\gamma$ is divided on $\gamma_{p}$ and $\gamma_{\mathrm{t}}$ angles of frames and how does turning resistances are changed to due increasing wheel loaders bucked mass and type of ground. 


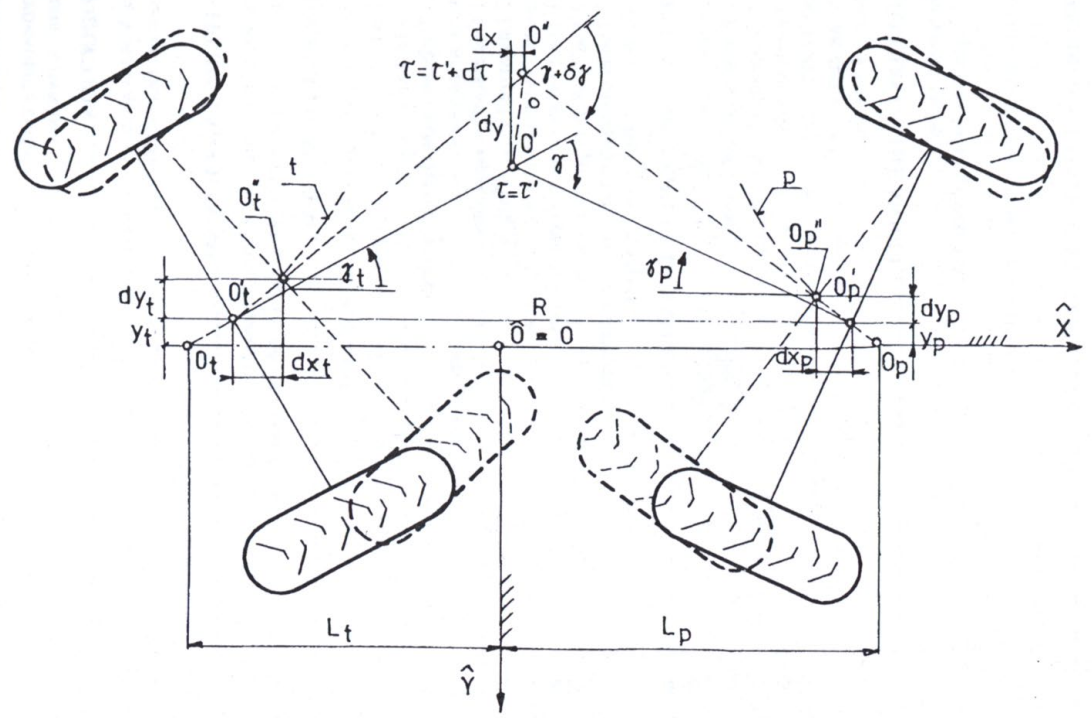

Fig. 1. The turning position of an articulated vehicle and shift trajectories $p, t, o[1]$

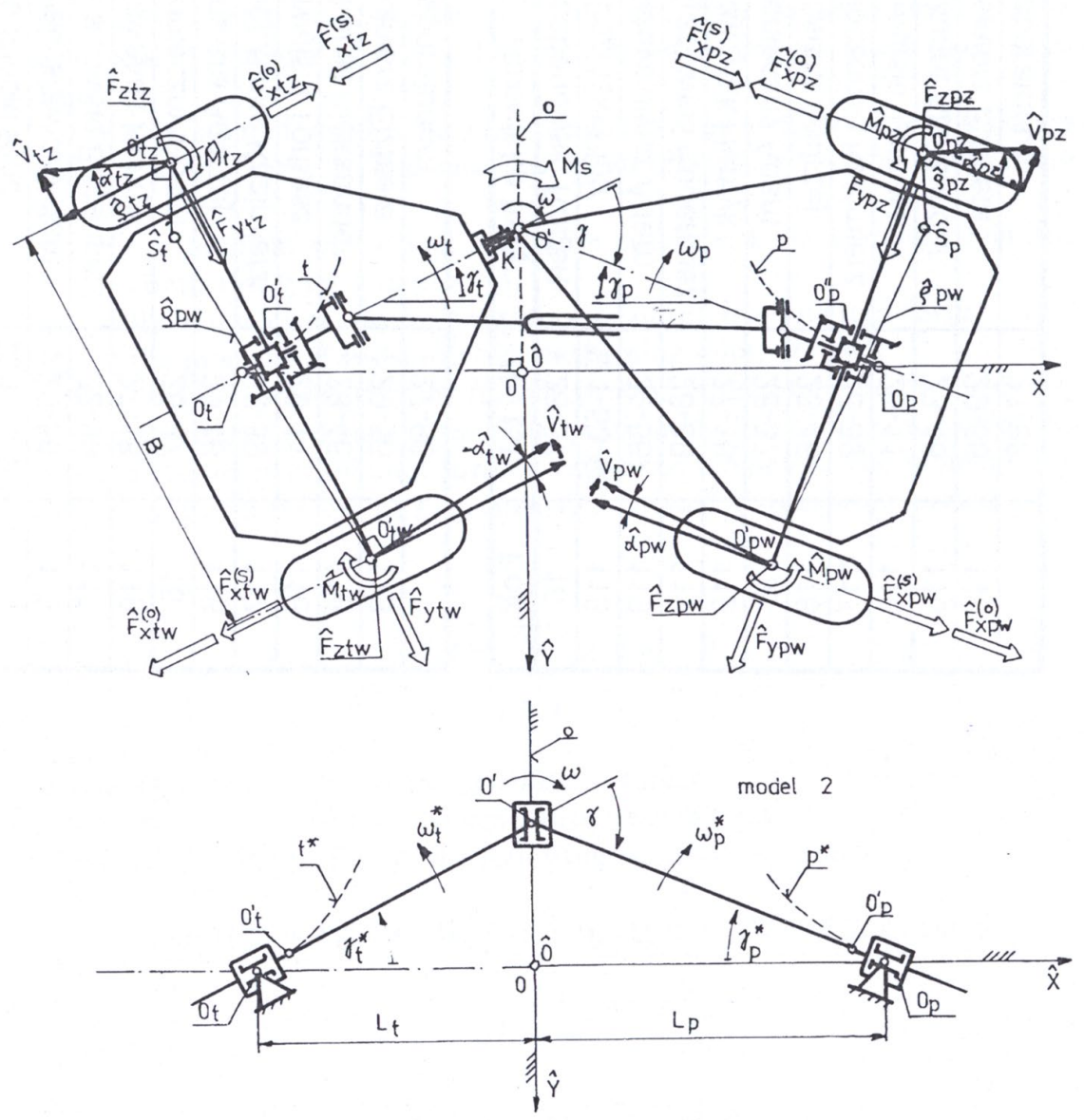

Fig. 2. Steering kinematics while symmetrical turning resistances of frames [1] 


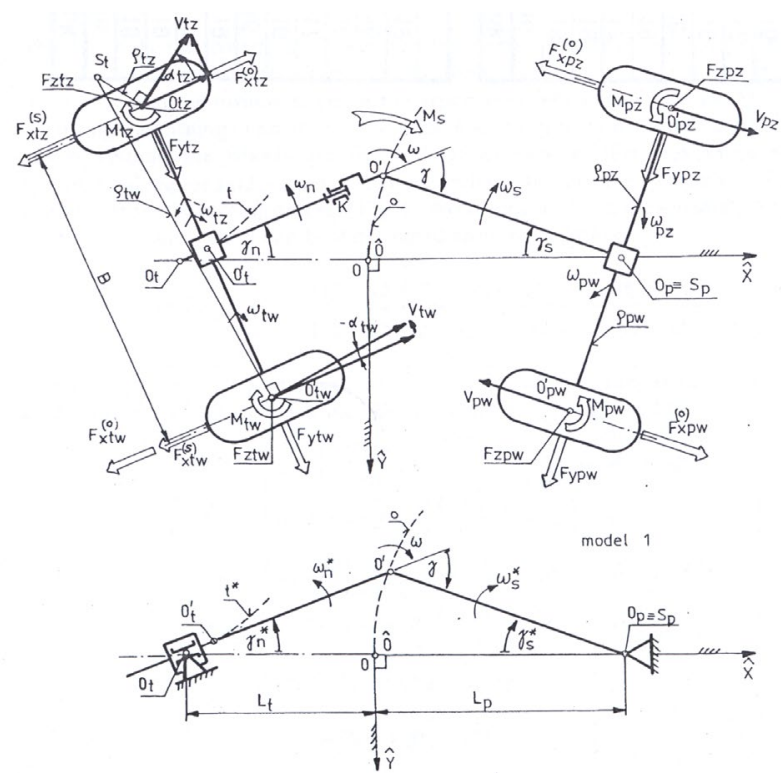

Fig. 3. Steering kinematics while unsymmetrical turning resistances of frames [1]

Identify of an exact mechanism of dividing an angle $\gamma$ to $\gamma_{\mathrm{p}}$ and $\gamma_{\mathrm{t}}$ allows for preparing a control system for traction control [3] and mitigate an effect of articulated vehicles snaking phenomena [4].

\section{Test method and measuring stand}

The experiment was conducted on mini wheel loader L052 Fadroma, overall mass m=1470 kg. The vehicle drives all 4 wheels by using a hydrostatic drive. The driving system consists of a hydraulic pump, valves and two hydraulic motors. Each hydraulic motor drives a differential mechanism without a lock to drive wheels. Basic mass and geometry parameters are shown in Fig. 4.

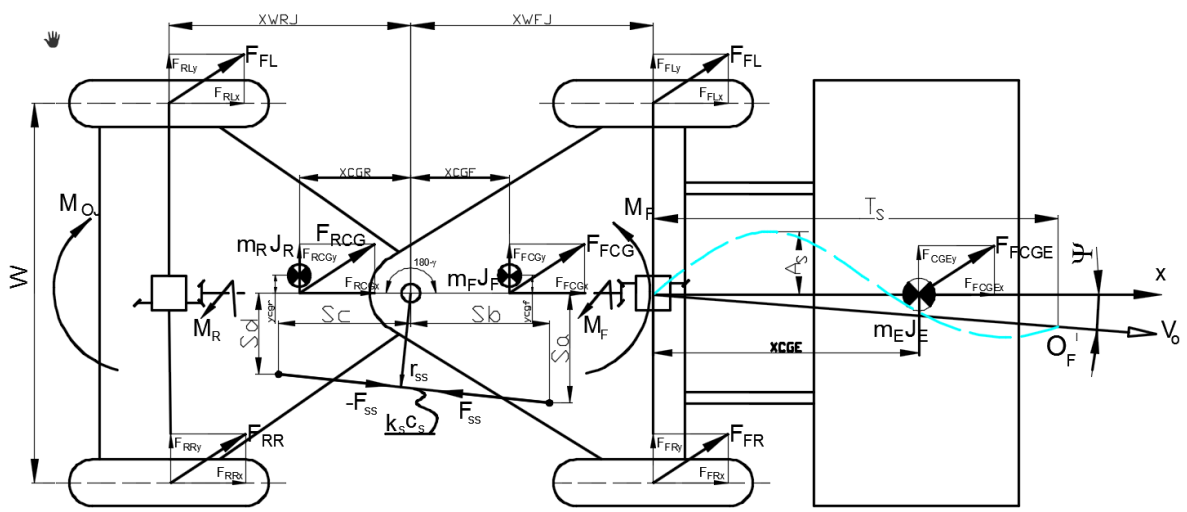

Fig. 4. The basic geometry and mass parameters of mini wheel loader $L 052, X_{c g r}=0,8 ; Y_{c g r}=0,2$;

$$
\begin{gathered}
X_{c g f}=0,7 ; Y_{c g f}=0,1 ; X_{w r j}=1,3 m ; X_{w f j}=1,4 ; m_{r}=780 \mathrm{~kg} ; m_{f}=690 \mathrm{~kg} ; m_{r f}=300 \mathrm{~kg} ; m_{l f}=390 \mathrm{~kg} ; \\
m_{r r}=430 \mathrm{~kg} ; m_{r l}=350 \mathrm{~kg}
\end{gathered}
$$

A measuring system consists of 3-axis the Earth magnetic field sensor HMC5883L and 3-axis acceleration sensor ADXL345. Modules were connected to microcontroller system by I2C protocol then the data send by RS232 protocol to Matlab/Simulink software. During the measurements of turning angles of frames by the use of the Earth field magnetic sensor, there is a 
need to compensate incline of a module and an acceleration sensor was used to compensate results. Measurement uncertainty is estimated to $\pm 0,5^{\circ}$ but it strongly depends on environmental the experiment takes place. Author believes that such accuracy is sufficient. In the Matlab/Simulink compensation model correction were calculated by following equations:

$$
\begin{aligned}
& \operatorname{tg}(\text { roll })=\frac{A_{y}}{A_{z}} \\
& \operatorname{tg}(\text { pitch })=\frac{-A_{x}}{A_{y} \sin (\text { roll })+A_{z} \cos (\text { roll })} \\
& \operatorname{tg}(\text { yaw })=\frac{\left(M_{z}-K_{z}\right) \sin (\text { roll })-\left(M_{y}-K_{y}\right) \cos (\text { roll })}{\left(M_{x}-K_{x}\right) \cos (\text { pitch })+\left(M_{y}-K_{y}\right) \sin (\text { pitch }) \sin (\text { roll })+\left(M_{z}-K_{z}\right) \sin (\text { pitch }) \cos (\text { roll })}
\end{aligned}
$$

where: $\quad A_{x}, A_{y}, A_{z}$ - measured acceleration, $M_{x}, M_{y}, M_{z}$ - measured angle orientation, $\mathrm{K}_{\mathrm{x}}, \mathrm{K}_{\mathrm{y}}, \mathrm{K}_{\mathrm{z}}$ - the Earth magnetic field sensor calibration coefficient, roll, pitch, yaw - calculated correction angle.

In the literature [6], [7], [8] a similar compensation method was used and the measuring method was performed with good precision.

Experiments were conducted on two different friction coefficient of the ground. During the first set of measurements friction coefficient was about $\mu=0.62$ for all wheels and next, only the front wheels were situated on $\mu=0.25$. During experiment bucket load was changing form $\mathrm{m}_{\mathrm{E}}=0$ to $300 \mathrm{~kg}$. Near right front wheel on front axle symmetry line, a measuring module was mounted. A potentiometer transducer situated between the front and rear frame measures articulation angle. The pressure sensor in a hydraulic cylinder was measuring turning resistance torque. Experimental result while turning with $300 \mathrm{~kg}$ load in a bucket and $\mu=0.62$ ground friction coefficient is shown in Fig. 5.

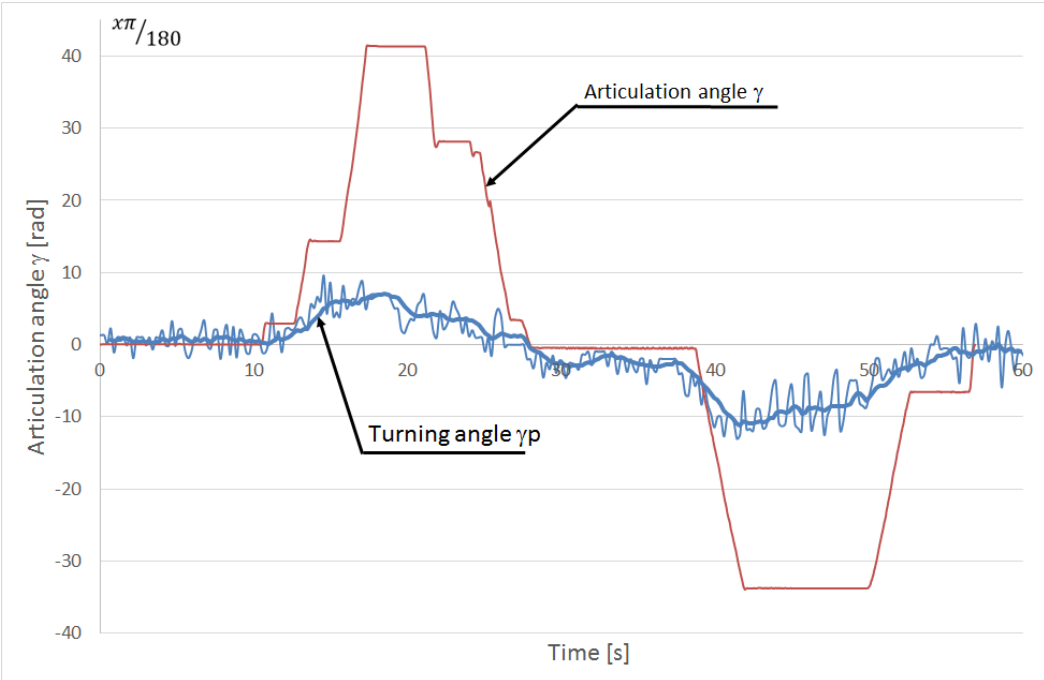

Fig. 5. Changing of $\gamma_{p}$ as a result of a vehicle turning $\gamma$ on a ground with a friction coefficient of $\mu=0.62$ and bucket mass $m_{E}=300 \mathrm{~kg}$ 
The experiment results showed higher values for a turning angle $\gamma_{\mathrm{p}}$ for clockwise rotation to turning the opposite direction for friction coefficient of $\mu=0.62$. We can observe that the ratio $\gamma / \gamma_{p}$ for clockwise rotation obtains $\gamma / \gamma_{p} \approx 3$ when anticlockwise turning coefficient gets $\gamma / \gamma_{p} \approx 5$. This is made probably by unsymmetrical normal reaction of the wheels on the right and left side per driving axle. This result in different rolling resistant torque of each wheel.

For ground friction coefficient of $\mu=0.25$, an asymmetry diminishes and the ratio is obtaining values $\gamma / \gamma_{\mathrm{p}} \approx 4$ in both turning directions. This is because a side slip of front wheels makes longitudinal and lateral forces in tire-ground contact becomes smaller and in results, lower braking torques were acting on wheels.

Results obtained during the experiment for different load and friction coefficient of ground have a similar plot. Maximum values of $\gamma_{p}$ and $\gamma$ are presented in Fig. 6.

Results showing how bucket load affects turning resistance force $\mathrm{F}_{\mathrm{s}}$ are presented in Fig. 7.

Experimental results showed that on the ground with friction coefficient $\mu=0.62$ turning resistance is rising with bucket mass. This is because of uneven normal reactions of front $\mathrm{m}_{\mathrm{f}}=690 \mathrm{~kg}$ and rear axle $\mathrm{m}_{\mathrm{r}}=780 \mathrm{~kg}$. We can observe that turning resistance rises fast until normal reactions on a front and rear wheels gets equal values. Beyond this point, the rear frame is going up because of a high load in a bucket. This result in a situation, that normal reaction on rear wheels becomes smaller and of course, they are losing the capability of putting high lateral and longitudinal forces. This results in obtaining smaller turning resistance forces with a high load in a bucket. Lowering a friction coefficient $\mu=0.25$ is giving similar results but with smaller resistance forces.

\begin{tabular}{|c|r|r|r|l|l|}
\hline $\begin{array}{l}\text { Mass } \\
\mathrm{m}_{\mathrm{E}}[\mathrm{kg}]\end{array}$ & $\begin{array}{l}\text { Coef. } \\
\mu[-]\end{array}$ & $\begin{array}{l}\text { Angle } \gamma \\
{[\mathrm{deg}]}\end{array}$ & $\begin{array}{l}\text { Angle } \gamma_{\mathrm{p}} \\
{[\mathrm{deg}]}\end{array}$ & $\begin{array}{l}\text { Sum } \gamma \\
{[\mathrm{deg}]}\end{array}$ & $\begin{array}{l}\text { Sum } \gamma_{\mathrm{p}} \\
{[\mathrm{deg}]}\end{array}$ \\
\hline 0 & 0.62 & -34 & -11.2 & & \\
0 & 0.62 & 40 & 8 & 74 & 19.2 \\
\hline 100 & 0.62 & -32 & -7 & & \\
100 & 0.62 & 41 & 12 & 73 & 19 \\
\hline 200 & 0.62 & -32 & -10.5 & & \\
200 & 0.62 & 41 & 7.25 & 73 & 17.75 \\
\hline 300 & 0.62 & -34 & -11 & & \\
300 & 0.62 & 41 & 7.9 & 75 & 18.9 \\
\hline & & & & & \\
\hline 0 & 0.25 & -32 & -9 & & \\
0 & 0.25 & 38 & 10 & 70 & \\
\hline 100 & 0.25 & -32 & -8 & & \\
100 & 0.25 & 40 & 12 & 72 & \\
\hline 200 & 0.25 & -35 & -8 & & \\
200 & 0.25 & 42 & $5(?)$ & 77 & \\
\hline 300 & 0.25 & -30 & -10 & & \\
300 & 0.25 & 40 & 12 & 70 & \\
\hline
\end{tabular}

Fig. 6. Experimental investigation results for different mass and ground friction coefficient 


\begin{tabular}{|c|c|c|c|c|}
\hline $\begin{array}{c}\text { Mass } \\
\mathrm{m}_{\mathrm{E}}[\mathrm{kg}]\end{array}$ & $\mu(1)[-]$ & $\mathrm{F}_{\mathrm{s}}(1)[\mathrm{N}]$ & $\mu(2)[-]$ & $\mathrm{F}_{\mathrm{s}}(2)[\mathrm{N}]$ \\
\hline 0 & 0.62 & 3780 & 0.25 & 4390 \\
100 & 0.62 & 4300 & 0.25 & 4400 \\
200 & 0.62 & 4970 & 0.25 & 4600 \\
300 & 0.62 & 5427 & 0.25 & 4573 \\
\hline
\end{tabular}

Fig. 7. Turning resistance force with different bucket mass and ground friction coefficient

\section{Conclusions}

Presented measuring method can be used to measure turning angles of vehicles frames. Measuring accuracy depends on environmental conditions because the Earth magnetic field sensor is used. Using the acceleration sensor for the Earth magnetic field sensor tilt module compensation works effectively and increases the precision of measuring device. Estimated error is about $\pm 0,7^{\circ}$ in walls of the laboratory. The measurements were performed at a standstill and no additional acceleration than a tilting sensor module was detected. A dynamic impact can influence temporary significant error in angle orientation measurement because of incorrect calculation of a tilt correction value.

The results showed that unsymmetrical normal reactions of wheels, mass in the bucket, friction coefficient have a significant effect on the turning angle of frames and turning resistance torque. At a high tires friction coefficient, an asymmetry rises because of higher resistant torques at a machine side. Lower friction coefficient allows for easier rolling of tires and obtaining symmetry of $\gamma_{p}$ and $\gamma_{t}$ while turning. Higher the bucket mass at a first phase, increases steering torque but at a second phase it decreases. This is because the overweighting machine over the front tires and lowering the normal reactions at rear tires as a result.

Adding of a second measuring point on a rear frame allows for measuring trajectory, measuring with higher precision $\gamma_{\mathrm{p}}$ and $\gamma_{\mathrm{t}}$, to propose snaking phenomena control systems and driver assist systems.

\section{References}

[1] P. Dudzinski, Problems of turning process in articulated terrain vehicles, Journal of Terramechanics, ISTVS, vol 19, No. 4, p.246-256, 1983. https://doi.org/10.1016/00224898(83)90030-7

[2] P. Dudzinski, Lenksysteme für Nutzfahrzeuge, Springer-Verlag, 2006

[3] A. Skurjat, A. Kosiara, Directional stability control of body steer wheeled articulated vehicles. W: Dynamical Systems in Applications, Łódź, Poland, December 11-14, 2017, Springer, cop. 2018. s. 363-371. https://doi.org/10.1007/978-3-319-96601-4_33

[4] P. Dudziński, A. Skurjat, Research on the influence of geometric parameters on the phenomenon of snaking of articulated vehicles. Zeszyty Naukowe - Wyższa Szkoła Oficerska Wojsk Lądowych im. gen. T. Kościuszki. 2017, t. 186, vol. 49, nr 4. https://doi.org/10.15804/athena.2016.50.04

[5] P. Dudziński, G. Hapel, A. Skurjat, Patent. Polska, nr 216468. Sposób i urządzenie do automatycznego adaptacyjnego sterowania pojazdem nr 388851, 2014 
[6] S. Madgwick, Automated calibration of an accelerometers, magnetometers and gyroscopes A feasibility study, 2010

[7] Pablo Esteban Quiroga Garcia Wenjie Li, On Indoor Positioning for Mobile Devices, Department of Signals and Systems, Chalmers University of Technology, Goteborg, Sverige, 2011

[8] C. Treffers, L. Wietmarschen, Position and orientation determination of a probe with use of the IMU MPU9250 and a ATmega328 microcontroller, TuDelft, 2016 